\title{
Pulp Regeneration by Harnessing Dental Pulp Stem Cells
}

\author{
Misako Nakashima and Koichiro lohara \\ Additional information is available at the end of the chapter
}

http://dx.doi.org/10.5772/58905

\section{Introduction}

Teeth possess multiple functions. Besides mastication, they are important for taste, tactile sense, speech and esthetics. The longevity of teeth is critical for the maintenance and enhancement of quality of life. Over fifty percent of extracted teeth are caused by caries and root fracture. Deep caries and pulp exposure have been treated by pulp capping or partial pulp amputation to preserve pulp tissue, with limited success. In case of irreversible pulpitis, the entire pulp has to be removed and the root canal space has to be completely filled with artificial materials after disinfection. However, incomplete disinfection and coronal leakage cause periapical disease. The endodontic success rate is less than $50-70 \%$ for retreatment of those endodontically failed teeth with periapical lesions and/or some clinical symptoms. Enlargement and debridement of infected root canals render teeth brittle. The post-operative procedures with post preparation also increase the possibility of root fracture. Therefore, loss of pulp vitality is highly associated with root fracture and tooth loss. There has been no superior synthetic material to replace natural pulp and dentin. Thus, regenerating pulp-dentin tissues seems to be an ideal approach to restore tooth functions compromised by pulp injury and/or inflammation (Nakashima and Akamine, 2005; Nakashima, et al., 2009). Under natural conditions, pulp and dentin have limited capacity to regenerate and repair when damaged. This chapter describes recent advances and promising approaches for pulp regeneration, including isolation and characteristics of pulp stem/progenitor cells and partial and complete regeneration of pulp. The complete pulp regeneration with other tissue stem cells is also addressed. 


\section{Pulp regeneration in mature teeth with complete apical closure}

\subsection{Pulp stem/progenitor cells suitable for pulp regeneration}

Dental pulp tissue is rich in vasculature and innervation. There is an intimate association of innervation with vasculature of the dental pulp, involving in pulp homeostasis. Thus, angiogenesis and re-innervation are critical prerequisites for pulp regeneration (Nakashima and Akamine, 2005). Numerous studies have reported the isolation of adult pulp stem/ progenitor cells with high proliferative and multi-differentiation potential in vitro. However, only a few studies have demonstrated angiogenesis and re-innervation in vivo after transplantation.

Porcine and human pulp CD31'side population(SP) cells and CD105/endoglin ${ }^{+}$cells, positive for CD29, CD44, CD73, and Thy-1/CD90, and negative for CD31, CD45 and CD133, increase the blood flow including high density of capillary formation after transplantation into mouse hindlimb ischemia (Iohara et al., 2008; Nakashima et al., 2009). SP cells are enriched in the 'true' or 'mother' adult stem cells, which exhibite lower level of the DNA binding fluorescent dye, Hoechst 33342than the rest of pulp cells. CD105/endoglin is a component of the transforming growth factor-beta receptor complex. CD105 is widely expressed on mesenchymal stem cells (MSCs), and the positive isolation strategy based on the selection of the cells that express CD105 has been proposed for isolation of MSCs. Pulp CD31'cells and CD105+cells induce angiogenesis and neurogenesis in rat cerebral ischemia by releasing neurotrophic factors, such as VEGF in peri-infarct area and promoting migration and differentiation of the endogenous neuronal progenitor cells, and accelerate the functional recovery (Sugiyama, et al., 2011). Local transplantation of these pulp stem/progenitor cells results in successful engraftment in proximity to the newly formed vasculature without direct incorporation into vessels, suggesting enhancement of angiogenesis and re-innervation by trophic effects (Iohara, 2008; Sugiyama, 2011). The conditioned medium of pulp stem/progenitor cells contain high concentration of angiogenic/neurotrophic factors such as brain-derived neurotrophic factor (BDNF), nerve growth factor (NGF), vascular endothelial growth factor (VEGF-A), glial cell-line derived neurotrophic factor (GDNF), granulocyte-macrophage colony-stimulating factor (GM-CSF), granulocyte colony-stimulating factor (G-CSF), matrix metalloproteinase 3 (MMP3). These factors have effect on proliferation, migration, and anti-apoptosis (Iohara, 2008, Sugiyama, 2011). On the other hand, unfractionated total pulp cells express lower level of angiogenic/ neurotrophic factors, and are less effective on angiogenesis and neurogenesis compared to CD31-SP cells and CD105 ${ }^{+}$cells after transplantation in hindlimb ischemia and brain ischemia (Nakashima, et al., 2009). Thus, potential utility of some subfractions of pulp stem/progenitor cells has been demonstrated as cell sources for cell-based therapy of angiogenesis, re-innervation and pulp regeneration. MSCs secrete soluble cytokines and growth factors that function in a paracrine fashion, contributing to repair and regeneration by enhanced cell survival, angiogenesis, neurogenesis and neuroprotection, and by activating endogenous stem cells (Kassis et al., 2011). 


\subsection{Partial pulp regeneration with pulp stem/progenitor cells}

Recent advances in tissue regeneration have led to the possibility of partial pulp regeneration in case of pulp exposure or pulpotomy of partial pulptitis. There are two methods in partial pulp regeneration even in mature teeth with complete apical closure; (i)transplantation of pulp stem/progenitor cells with scaffold or engineered pulp tissue, and (ii) application of migration/ homing factors with scaffolds.

The first method (Figure 1A) is evidenced by an experiment in which canine autologous pulp CD31-SP cells are transplanted in the cavity on the amputated pulp one day after three dimensional cultures with collagen scaffold. The cavity on the amputated pulp is filled with the pulp tissue with well-developed vasculature and innervations (Figure 2A, C-E). The tubular dentin is formed along the dentinal wall (Figure 2F, G) (Iohara, et al., 2009). Many of transplanted CD31-SP cells are in the proximity of the newly formed capillaries and express angiogenic/neurotrophic factors, implicating trophic effects on neovascularization (Iohara et al., 2009). On the other hand, after transplantation of CD31+SP cells, fewer capillaries and lower volume of the regenerated tissue are seen in the cavity on the amputated pulp compared with transplantation of CD31-SP cells (Figure 2C)(Iohara, et al., 2009), suggesting different potential utility in induction of pulp regeneration among subfractions of pulp stem/progenitor cells.

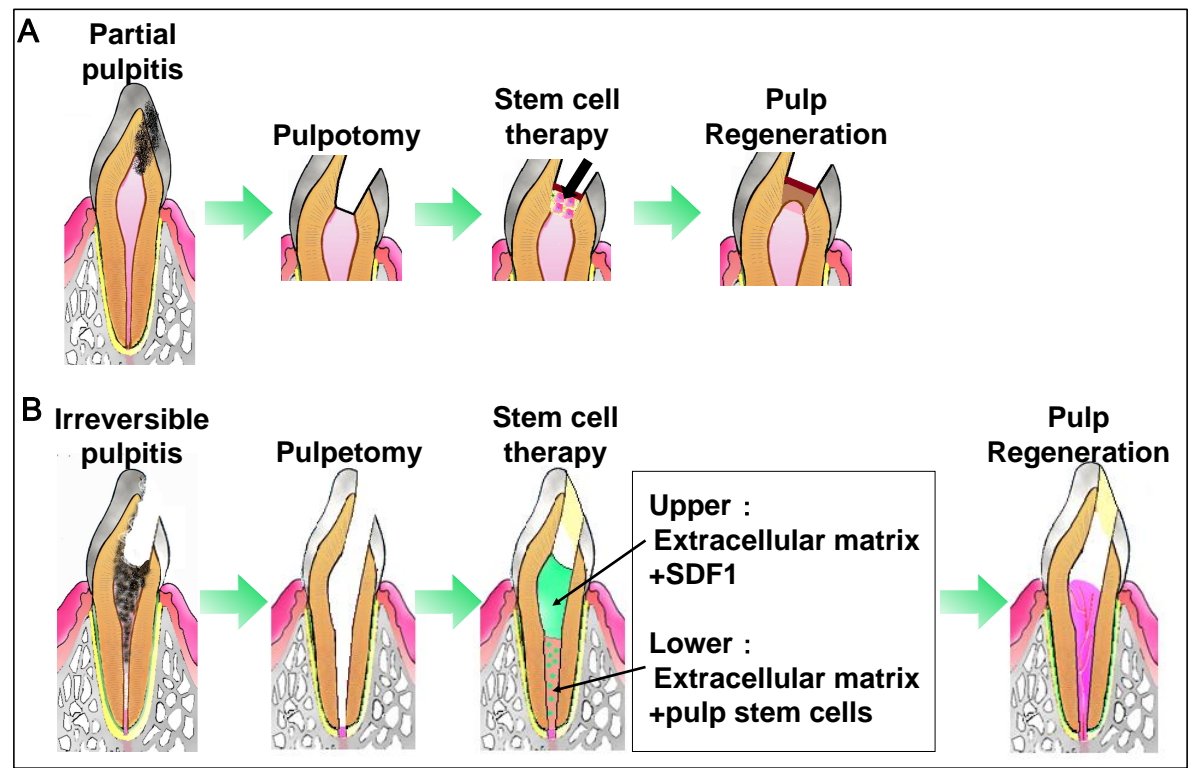

Figure 1. Schematic diagrams of a canine model for partial and complete pulp regeneration in permanent mature teeth. (A) Partial pulp regeneration model. (B) Complete pulp regeneration model. (lohara et al., Tissue Eng. 2011) 

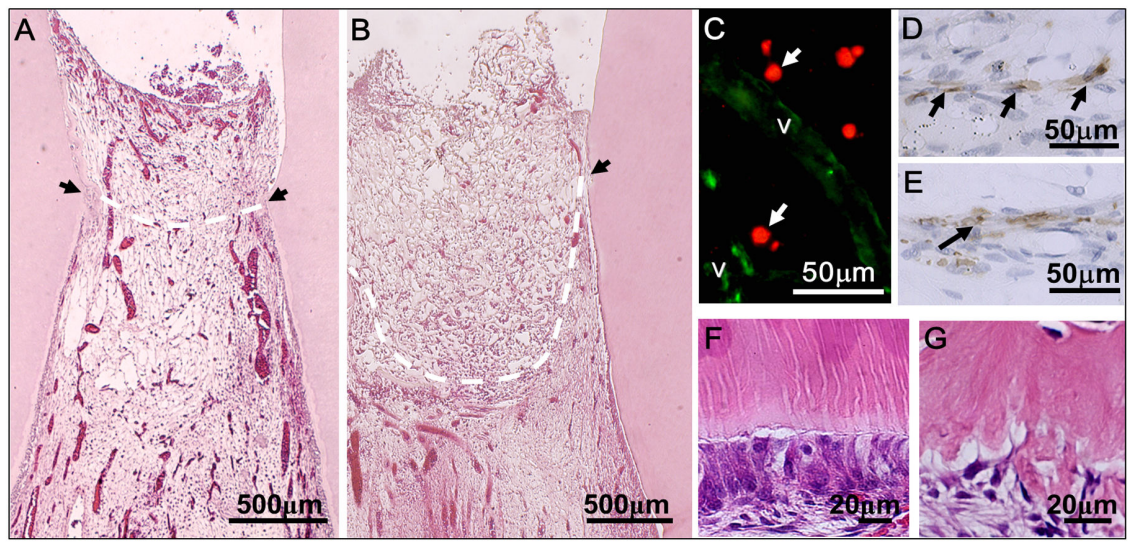

Figure 2. Neovascularization and pulp regeneration in the cavity on the amputated pulp of dogs after autogenous transplantation of CD31\%CD146 SP cells. (A, B) Fourteen days after transplantation of a three-dimensional pellet on day 1 of culture with type I and type III collagen. H.-E. staining. Amputated sites of dental pulp are indicated by arrows. The established pulp tissue is marked by dotted lines. (A) CD31\%CD146-SP cells. The cavity in the amputated pulp is filled with regenerated pulp tissue. Capillaries extended to the top of the tissue beneath the site of the filling cement. (B) CD $31^{+} /$CD146-SP cells. Few capillaries are seen in the cavity on the amputated pulp despite engraftment of the transplanted pellet. (C) Immunostaining of CD146. Dil-labeled CD31\%CD146-SP cells (arrows) can be seen close to the newly formed capillaries (V). (D, E) Immunostaining of neurofilament (arrows).(D) in the regenerated pulp tissue on the amputated site. (E) in the normal pulp tissue under the amputated site. (F) Tubular dentin formation along the dentinal wall in the cavity (G) Osteodentin formation at the top of the cavity under cement. (Iohara et al., Reg Med. 2009)

\subsection{Complete pulp regeneration with pulp stem/progenitor cells and migration factors}

Complete pulp regeneration is optimistically considered by transplantation of autologous pulp stem/progenitor cells in case of irreversible pulpitis of even mature teeth with complete apical closure. The transplanted cells should survive with nutrition and oxygen in the pulpectomized root canals before revascularization. Some subfractions of pulp stem/progenitor cells, such as CD105+cells or CD31-SP cells, have more regenerative potential compared with unfractionated total pulp cells. Pulp stem/progenitor cells are capable to induce angiogenesis and re-innervation without direct incorporation into vasculature. Thus, the induction of MSC recruitment from surrounding tissues or from the circulation by migration/homing factors together with transplanted stem/progenitor cells can be a helpful modality to initiate and support cell therapy for pulp regeneration. Pulp CD31-SP cells and CD105 ${ }^{+}$cells are CXCR4-positive, having high migratory and proliferative activities with SDF-1 (Iohara, et al., 2008; Nakashima, et al., 2009). Thus, one candidate for migration/homing factors is SDF-1, a chemokine for CXCR4-positive stem cells. It is plausible that SDF-1 transplanted with pulp stem cells provides signals for homing and proliferation of endogenous stem cells originating from surrounding periodontal ligament, bone marrow and vasculature.

Optimal scaffolds should also be developed for efficient and safe complete pulp regeneration. It is critical for the scaffold to be injected into the root canal with optimal flow, devoid of bubble 
formation and mechanical properties after hardening in vivo. In addition the scaffold has to be biocompatible, with high bioactivity to integrate trophic factors secreted by stem cells. Furthermore it should be biodegradable by releasing these factors to replace the damaged tissue by newly regenerated tissue without immune response. In addition, the scaffold should not stimulate mineralization and differentiation of odontoblasts and/or osteodentinoblasts in the root canal except along the dentinal wall for pulp regeneration (Nakashima, et al., 2009). Potential scaffolds include natural polymers, such as collagen and gelatin with good biocompatibility and bioactivity (Nakashima and Akamine, 2005).

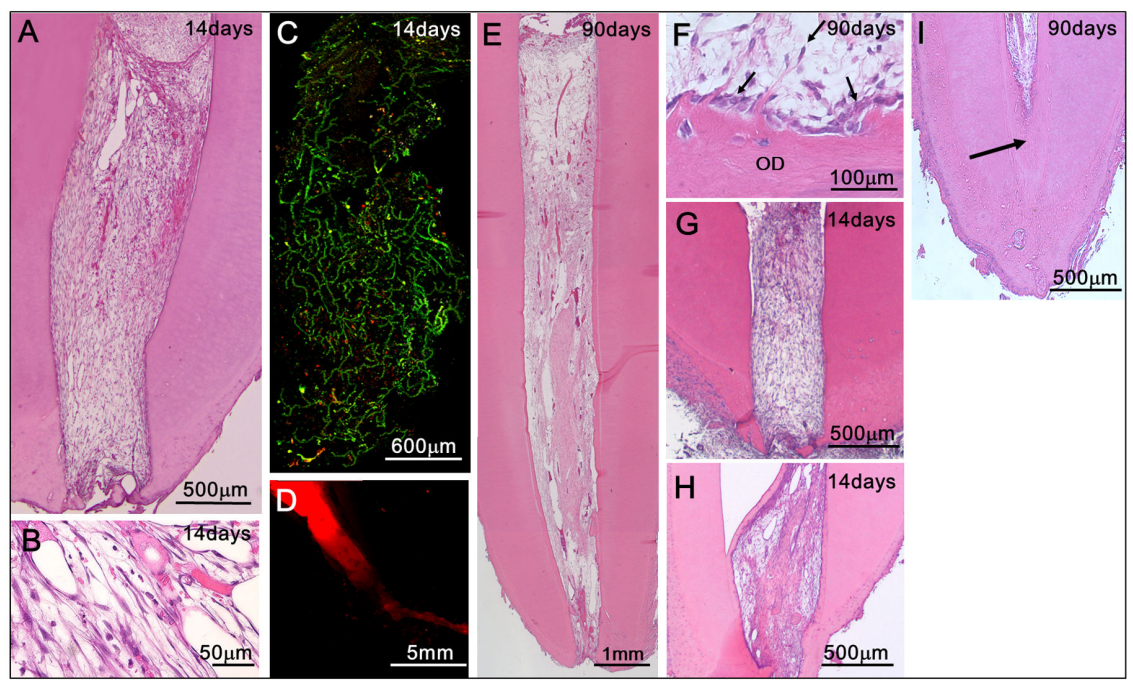

Figure 3. Complete pulp regeneration after autologous transplantation of CD $105^{+}$cells with SDF- 1 in the pulpectomized root canal in dogs. (A-F) CD $105^{+}$cells with SDF-1. (C) Three dimensional images of new vascularization by whole mount immunostaining with lectin. (D) Dil-labeling from the upper part of the regenerated pulp on day 14. Note inferior alveolar nerve connecting to the regenerated pulp, suggesting re-innervation. (F) Odontoblastic cells (arrows) lining to newly formed osteodentin/tubular dentin (OD) along with the dentinal wall. (G) CD105+cells only. (H) SDF-1 only. (I) Total pulp cells with SDF-1. Mineralized tissue (arrow) and osteodentin. (lohara et al., Tissue Eng. 2011)

Thus, based on the concepts described above, pulp regeneration has been demonstrated by using the triad, stem/progenitor cells, migration/homing factors and optimal scaffolds (Nakashima, et al., 2011, Iohara, et al., 2011)(Figure 1B). Pulpectomy with enlarged apical portion, $0.6 \sim 0.7 \mathrm{~mm}$ in width, was performed in mature teeth with complete apical closure in an experimental model in dogs (Iohara, et al., 2011, Ishizaka et al., 2012). Current evidence shows that the root canal is filled with pulp-like loose connective tissue with vasculature and nerves after autologous transplantation of pulp stem/progenitor cells, CD31-SP cells or pulp CD105 ${ }^{+}$cells, $5 \times 10^{5}$ cells in cell number, with SDF- 1 in collagen type I and type III scaffold (Figure 3A-F) (Nakashima, et al., 2011, Iohara, et al., 2011, Ishizaka et al., 2012). The three dimensional image of induced vascularization in the regenerated tissue (Figure 3C) is similar in density and orientation to those in the normal pulp. The neuronal process from regenerated 
pulp is connecting to the inferior alveolar nerve (Figure 3D)(Iohara et al., 2011). The transplanted pulp stem cells expressed angiogenic/neurotrophic factors, and localized in the vicinity of newly formed capillaries (Iohara et al., 2011; Ishizaka et al., 2012), suggesting potential trophic effects on angiogenesis. The odontoblast-like cells attached to the dentinal wall in the root canal, and produce dentin-like mineralized tissue extending their processes into dentinal tubules (Figure 3F). The enlarged apical portion following pulpectomy is filled by additional formation of dentin and cementum. On the other hand, transplantation of stem/ progenitor cells alone (Figure 3G), or SDF-1 alone (Figure 3H), yield significantly less pulp tissue. When unfractionated total pulp cells are implanted in place for the fractionated pulp stem cells, the regenerated tissue is significantly less in volume and undergo mineralization (Figure 3I) (Iohara et al., 2011). The regenerated tissue induced by the pulp stem/progenitor cells and SDF-1 is identical to be normal functional pulp tissue as demonstrated similar expression of the pulp tissue markers, Syndecan and TRH-DE mRNA. It is noteworthy that the qualitative and quantitative protein and mRNA expression patterns obtained by two dimensional electrophoretic analyses and microarray analyses are virtually identical (Iohara et al., 2011; Ishizaka et al., 2012). Furthermore, in a canine experimental model of periapical disease, which root canal is kept open for more than three weeks, pulp regeneration has been demonstrated after transplantation of pulp stem cells and SDF-1 as demonstrated in case of pulpitis. The possible mechanisms of pulp regeneration may involve the CXCR4/SDF-1 axis functioning as a migration/homing factor for CXCR4-positive endogenous stem cells to migrate to the coronal portion of the root canal, proliferate, and differentiate into endothelial cells for angiogenesis and re-innervation by angiogenic and neurotrophic factors secreted by transplanted stem cells. These findings suggest potential clinical translation of complete pulp regeneration by harnessing pulp stem/progenitor cells with high angiogenic/neurogenic potential and additional migration/homing factors in endodontic treatment. The mechanisms of recruitment and crucial molecules for cell migration are still unclear. Chemokines and their receptors which play critical roles need further scrutiny.

\subsection{Other sources of tissue stem cells for pulp regeneration}

Supply of autologous pulp tissue declines with age, and alternative sources of MSCs to pulp stem cells are necessary for clinical application in endodontic treatment. Some transcriptional and epigenetic analyses have revealed very similar profiles among a variety of MSCs from bone marrow, adipose tissue, placenta, umbilical cord and amnion (Aranda etal., 2009; Boeuf and Richter, 2010). Different expression profile of trophic factors and growth factors, however, has also been reported among MSC populations (Noël et al., 2008; Boeuf and Richter, 2010; Philippe et al., 2010). Thus, it is necessary to investigate the requirements and preconditions of MSCs for effective induction of pulp regeneration (Ishizaka, et al., 2012). A candidate of an alternative cell source for pulp regeneration is autologous bone marrow and adipose tissuederived MSCs due to neither ethical nor immunoreactive considerations. Transplantation of adipose CD31-SP cells resulted in formation of a similar amount of regenerated tissue compared with that of pulp CD31-SP cells (Figure 4A, C). Bone marrow CD31-SP cell transplantation induce significantly less amount of regenerated tissue compared with other two (Figure $4 \mathrm{~B}, \mathrm{D})$. Those regenerated tissues, however, are all identical to pulp tissue confirmed by 
morphological and functional similarity, mRNA expression patterns of microarray analyses and two-dimensional electrophoretic analyses (Ishizaka, 2012). Thus, it is suggested that MSCs from different sources are comparable in pulp regeneration in vivo and that their capabilities are not influenced by the niche of their origin. Enhanced matrix formation in adipose cell transplantation, which lead to root canal obliteration after longer period, suggest that some trophic factors, growth/differentiation factors and/or inhibitors of stemness may influence on active pathway for differentiation in adipose stem cells. Further studies are needed to elucidate its involved pathways and processes.
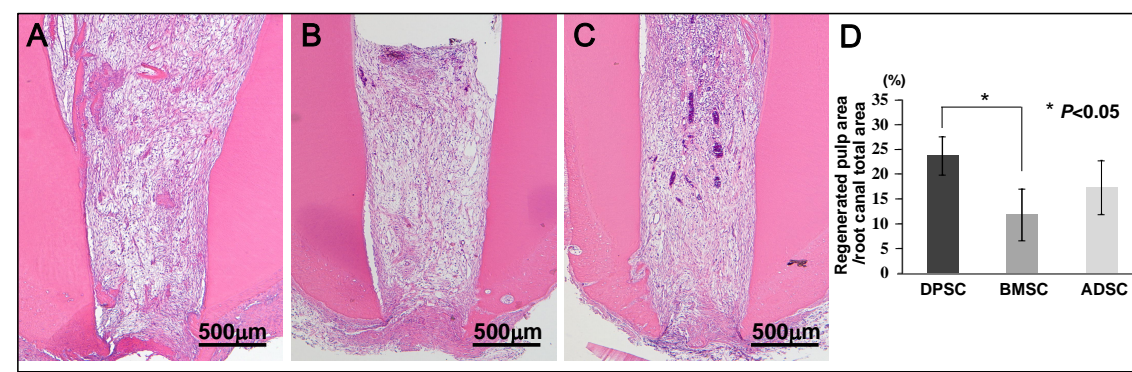

Figure 4. Complete regeneration of pulp tissue after autologous transplantation of CD31-SP cells with SDF-1 in the emptied root canal after pulpectomy in dogs on 14days. (A) pulp CD31-SP cell transplantation. (B) bone marrow CD31SP cell transplantation. (C) adipose CD31-SP cell transplantation. (D) Ratio of regenerated area to root canal area. Data are expressed as means \pm SD of 5 determinations. (Ishizaka et al., Biomaterials. 2012)

\section{Conclusion}

Cell therapy with subfractions of pulp stem/progenitor cells, CD31-SP cells or pulp CD105 ${ }^{+}$ cells is capable to induce pulp regeneration, presenting the following features: i) vascularization, ii) similar cell density and architecture of the extracellular matrix as the natural pulp, iii) new odontoblasts aligning with the dentinal wall to produce new dentin, and iv) innervation.

CD31-SP cells have to be labeled with DNA binding Hoechst 33342 and isolated by flowcytometry, which requires the confirmation of safety. CD105 cells have to be isolated by magnetic antibody beads method if not by flowcytometry, and have to be cost effective. The unfractionated total pulp cells by colony formation method are less suitable for pulp regeneration since the regenerated tissue is much less in volume and much more to undergo mineralized compared with fractionated stem/progenitor cells. A novel isolation method capable of confirmation of safety and efficiency from a small amount of pulp tissue should be developed prior to clinical trials. Although there are still some hurdles to overcome in regenerative endodontics, there are the rewards of immense clinical advantages and benefits for patients to maintain the function and longevity of teeth resulting in maintenance of whole body and enhancement of quality of life. 


\section{Acknowledgements}

This work was supported by Collaborative Development of Innovative Seeds, Potentiality Verification Stage from Japan Science and Technology Agency to MN; and the Research Grant for Longevity Sciences (21A-7) from the Ministry of Health, Labour and Welfare to MN.

\section{Author details}

Misako Nakashima and Koichiro Iohara

National Center for Geriatrics and Gerontology, Research Institute, Japan

\section{References}

[1] Aranda, P.; Agirre, X.; Ballestar, E.; Andreu, E.J.; Román-Gómez, J. \& Prieto, I. et al. (2009). Epigenetic signatures associated with different levels of differentiation potential in human stem cells. PLoS One 4(11):e7809.

[2] Boeuf, S. \& Richter, W. (2010). Chondrogenesis of mesenchymal stem cells: role of tissue source and inducing factors. Stem Cell Res Ther 1(4):31.

[3]

[4] Iohara, K.; Zheng, L.; Ito, M.; Ishizaka,R.; Nakamura, H. \& Into T. et al. (2009). Regeneration of dental pulp after pulpotomy by transplantation of CD31/CD146-side population cells from a canine tooth. Regen Med 4(3): 377-385.

[5] Iohara, K.; Imabayashi, K.; Ishizaka, R.; Watanabe, A.; Nabekura, J.; Ito, M.; Matsushita, K.; Nakamura, H. \& Nakashima, M. (2011). Complete pulp regeneration after pulpectomy by transplantation of CD105+ stem cells with stromal cell-derived factor-1. Tissue Eng Part A 17(15-16): 1911-1920.

[6] Ishizaka, R.; Iohara, K.; Murakami, M.; Fukuta, O. \&Nakashima, M. (2012). Regeneration of dental pulp following pulpectomy by fractionated stem/progenitor cells from bone marrow and adipose tissue. Biomaterials 33(7):2109-2118.

[7] Nakashima, M. \&Akamine, A. (2005). The application of tissue engineering to regeneration of pulp and dentin in endodontics. J Endod 31(10):711-718.

[8] Kassis, I.; Vaknin-Dembinsky, A. \& Karussis, D. (2011). Bone marrow mesenchymal stem cells: agents of immunomodulation and neuroprotection. Curr Stem Cell Res Ther 6(1):63-68. 
[9] Nakashima, M.; Iohara, K. \& Sugiyama M (2009). Human dental pulp stem cells with highly angiogenic and neurogenic potential for possible use in pulp regeneration. $\mathrm{Cy}$ tokine \& Growth Factor Reviews 20(5-6):435-440.

[10] Nakashima, M. \& Iohara, K. (2011). Regeneration of dental pulp by stem cells. Adv Dent Res 23(3): 313-319.

[11] Noël, D.; Caton, D.; Roche, S.; Bony, C.; Lehmann, S. \& Casteilla L., et al. (2008). Cell specific differences between human adipose-derived and mesenchymal-stromal cells despite similar differentiation potentials. Exp Cell Res 314(7):1575-1584.

[12] Philippe, B.; Luc, S.; Valérie, P.B.; Jérôme, R.; Alessandra, B.R. \& Louis, C.(2010). Culture and use of mesenchymal stromal cells in phase I and II clinical trials. Stem Cells Int 2010:503593.

[13] Sugiyama, M.; Iohara, K.; Wakita, H.; Hattori, H.; Ueda, M.; Matsushita, K. \& Nakashima, M. (2011). Dental pulp-derived CD31(-)/CD146(-) side population stem/ progenitor cells enhance recovery of focal cerebral ischemia in rats. Tissue Eng Part A 17(9-10):1303-1311. 
